\title{
Characterization of Dislocations Found in an Array at a Mixed Character Small Angle Boundary of a Cross Rolled and Annealed Aluminium Sample
}

\author{
M. Shamsuzzoha
}

School of Mines and Energy Development, University of Alabama, Tuscaloosa, AL 35487, USA

A mixed character small-angle boundary of arbitrary orientation is considered to form by the union of two crystals with a twisting and a tilting of the crystallographic axis at the boundary plane. Such a boundary is therefore can be constructed from dislocation arrays of which the requisite tilt and twist boundaries are composed. Experimental evidences based on the atomic structure of symmetric low-angle tilt boundaries $[1,2]$ reveal that tilt boundaries can be described in terms of a regular array of edge dislocations separated by a nearly perfect lattice. Bright field images taken along the twist axis of small angle twist boundaries [3] reveals that a regular cross grid of screw dislocation lines form the surface of the twist boundary. It is of interest to understand the arrangement dislocation at the grain boundary surface of mixed character small angle boundaries. In annealing of heavily deformed materials a number of mixed character small angle boundaries can form by methods that may involve either unification of two crystals or by grouping dislocations in regular arrays in pure lattices. The mixed character boundary given here is prepared from Al by this process.

. Figure 1 shows an HREM micrograph of a segment of the mixed character boundary. The boundary assumes (110) plane as its boundary plane, and contains regular arrays of dislocations which exhibit extra half plane along [010], [110] and [1-10] (as shown in Figure 1b). However, the bi-crystal presents in these micrographs exhibits atomic resolution image for grain $\mathrm{A}$ and lattice resolution image for grain $\mathrm{B}$. The reason for the phenomenon is that across the boundary the grain $\mathrm{A}$ and $\mathrm{B}$ assume a mutual tilt amounting to $8^{\circ}$ and $11^{\circ}$ across [001] and [100] of the bi-crystal (Figure 2). The grain A of the bi-crystal is aligned along the [001], and under the available resolution of the TEM reveals (001) atomic resolution image. Under this orientation of the bi-crystal the grain B can only exhibit lattice resolution along [100], and no lattice resolution along [010]. In such a reality, it is worth investigating the nature of the dislocations present at the boundary. The boundary upon view along [100] is likely to exhibit the presence of a $11^{\circ}$ tilt boundary on (100) plane that is common to both participating grains, and with the same resolution of the TEM should reveal atomic resolution image in both sides of the boundary. Such a tilt boundary can be described in terms of regular arrays of pure edge dislocations separated by nearly perfect lattice. The extra half planes associated with such an edge dislocation (in the $\{111\}$ of the grains) upon viewed along [100] should exhibit a projected extra half plane along [001], [010], [011] and [0-11] directions. If a dislocation of this boundary of the (100) lies normal and just below the boundary of Figure 1a and 1b, a continuity of lattice around the dislocation tends to keep lattices across the experimental boundary (that lies above the dislocation) to be continuous. Hence, in an effort to arrange them so as to match the normal crystal plane as closely as possible the atoms in the vicinity of the dislocation of the boundary of (100) assume positions that is likely to reveals atomic resolution image in the vicinity of a typical dislocation of the boundary of (001). Such lattice arrangement makes the dislocations in Figure $1 \mathrm{a}$ and $1 \mathrm{~b}$ to become edge dislocations. However, atomic resolution image around each of such edge dislocations extends to such a small region of the interface that can allow the related projected extra half plane to be viewed (Figure 1b) only along [010], [110] and [1-10].

References

[1] M Shamsuzzoha, David. J. Smith, P. A. Deymier, Script. Met. \& Mat. 24, (1990),1611.

[2] M J. Mills, Phil. Mag. A66, (1989), 47.

[3] K. N. T. C. Chen, C. H. Tung, T. T. Sheng, A. Ploessl, R. Scholz and U. Gosele, Phil. Mag. A80(4), (2000), 88 


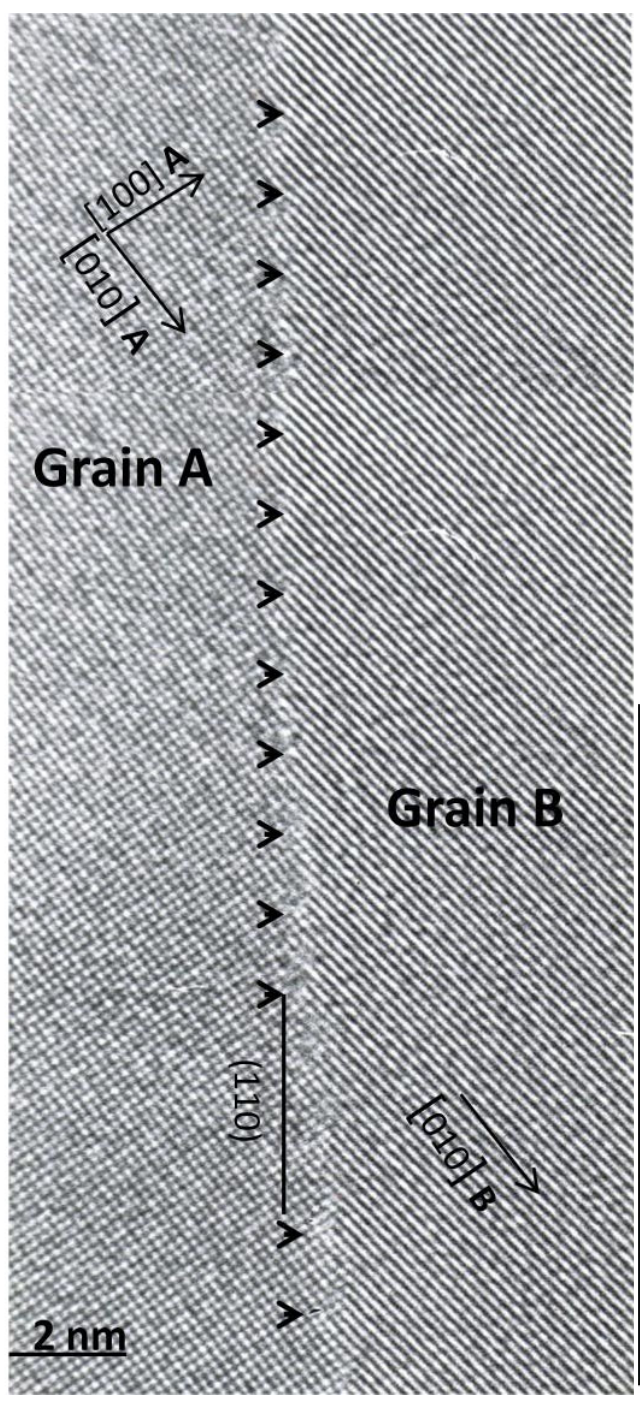

(a)

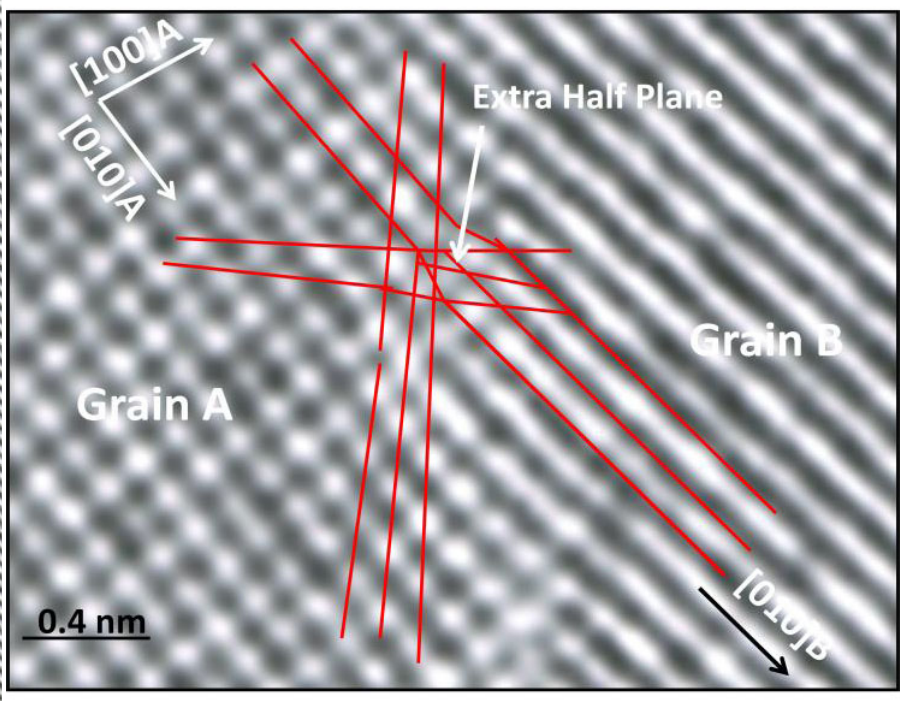

(b)

Figure 1. (a) HREM image recorded at $400 \mathrm{KeV}$ showing of a mixed character small angle boundary in $\mathrm{Al}$ sample with equally spaced dislocations marked by arrows. (b) Magnified image of a dislocation taken from (a). All the projected extra half planes associated with the dislocation are marked;

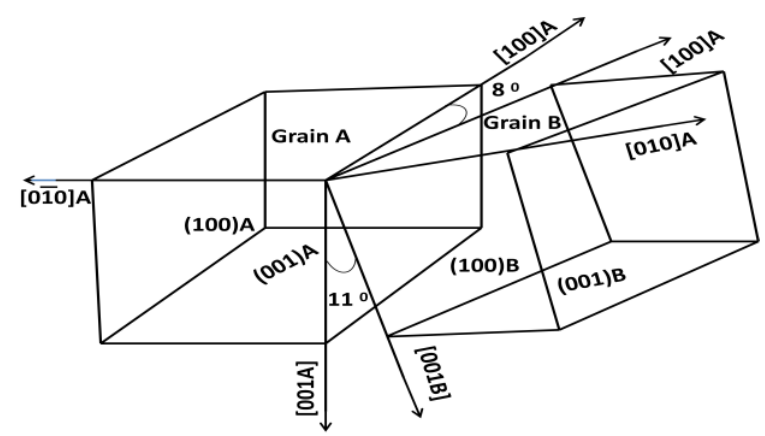

Figure 2. The schematic of the bi-crystal forming the grain boundary of Figure 1 